DOI: 10.12731/2658-6649-2020-12-3-80-93

УДК 796/799

\title{
ВЛИЯНИЕ УНИФИЦИРОВАННОЙ МЕТОДИКИ ПРЕПОДАВАНИЯ ФИЗИЧЕСКОЙ КУЛЬТУРЫ С ПРИМЕНЕНИЕМ СПОРТИВНЫХ НАСТОЛЬНЫХ ИГР НА ФУНКЦИОНАЛЬНОЕ СОСТОЯНИЕ ОРГАНИЗМА СТУДЕНТОВ СПЕЦИАЛЬНОЙ МЕДИЦИНСКОЙ ГРУППЫ
}

\begin{abstract}
Козлов А.В., Каткова А.М., Козлова М.А.
На сегодняиний день в системе научно-практических знаний остается малоизученным вопрос о развитии функционального состояния студентов с ОВЗ.

В статье рассматривается важная проблема здоровьесберегающих технологий средствами внедрения унифицированной методики с применением спортивных настольных игр в образовательный процесс студентов, отнесенных к специиальной медицинской группе.

Значимость исследования обусловлена тем, что данная унифицированная методика доступна для студентов при любых видах патологий, решает задачи инклюзивного образования, помогает увеличить двигательную активность, а как следствие и положительно влияет на функцчиональные показатели организма студентов специальных медицинских гpynn (CMГ).

В исследовании приняли участие 406 студентов (17-20 лет). Эксперимент состоял из 3 этапов. На каждом этапе проходило тестирование функционального состояния организма и велись наблюдения за динамикой показателей по Пробе Руфье и Индексу массы тела. Статистическая значимость различий полученных экспериментальных данных на 3 этапах эксперимента было проверено по: средней арифметической, приросту процентного показателя, U-критерию Манна-Уитни.

Проведенное исследование показало, что примененная методика подходит студентам с любыми видами отклонений в состоянии здоровья и улучиает их функииональное состояние организма.

Ключевые слова: здоровьесбережение; студенты специальных медицинских групп; инклюзивное образование; унифицированная методика; спортивные настольные игры; физическая культура.
\end{abstract}




\title{
INFLUENCE OF THE UNIFIED METHODOLOGY OF TEACHING PHYSICAL CULTURE WITH THE USE OF SPORTS BOARD GAMES ON THE FUNCTIONAL STATE OF THE ORGANISM OF STUDENTS OF A SPECIAL MEDICAL GROUP.
}

\author{
Kozlov A.V., Katkova A.M., Kozlova M.A.
}

Nowadays, in the system of scientific and practical knowledge, the question of developing the functional condition of students with HIA remains little studied.

The article considers the important problem of health-saving technologies by means of introducing a unified methodology with the use of sports board games into the educational process of students assigned to a special medical group. The significance of the study is due to the fact that this unified technique is available to students in all types of pathologies, solves the problems of inclusive education, helps to increase motor activity, and as a result, it positively affects the functional indicators of the body of students of special medical groups (SMG).

The study involved 406 students (17-20 years old). The experiment consisted of 3 stages. At each stage, the functional state of the condition was examined and the dynamics of the indicators for the Rufier Sample and Body Mass Index were monitored. The validity of the differences in the experimental data obtained at the 3 stages of the experiment was checked by: average arithmetic, percentage increase, Mann-Whitney U-criterion.

The study showed that the applied technique is suitable for students with any type of deviations in the state of health and improves their functional condition of the body.

Keywords: health care; students of special medical groups; inclusive education; unified methodology; sports board games; physical education.

\section{Введение}

В настоящий момент среди международного научного сообщества является актуальным вопрос влияния двигательной активности на функциональное состояние организма. [1,2,3]. Современные зарубежные исследования отмечают, что после поступления в высшие учебные заведения у молодых людей наблюдается гиподинамия $[4,5,6]$. Это несомненно отражается на функциональных показателях организма.

Данный вопрос особенно актуален для студентов с ограниченными возможностями здоровья (ОВ3). В образовательной среде их относят к 
специальной медицинской группе (СМГ), что приводит к возникновению новых задач в организации процесса обучения.

В последние годы в зарубежных источниках все чаще пишут про инклюзивное образование, которое вносят свои коррективы и в образовательную деятельность по физической культуре $[7,8,9,10]$. Отечественные ученые и преподаватели в области физической культуры тоже пытаются решить проблему инклюзивного образования [11]. Требования для студентов, отнесенных к основной медицинской группе, не применимы к категории студентов с ОВ3, как и в физкультурно-образовательном процессе, так и в оценивании $[12,13,14]$.

В настоящее время не проводилось научных исследований, где бы были бы рассмотрены результаты внедрения в образовательную программу унифицированной методики с блоком спортивных настольных игр и отслежена динамика показателей функционального состояния организма студентов СМГ.

Разработанная методика направлена на развитие физических способностей и функционального состояния организма.

Цель исследования заключается в изучении влияния унифицированной методики с применением спортивных настольных игр на функциональное состояние организма студентов специальных медицинских групп (СМГ).

Цель исследования структурировала следующие задачи:

1. Изучить отечественную и зарубежную научную литературу по вопросу поиска новых форм преподавания физической культуры студентам специальной медицинской группы;

2. Изучить показатели функционального состояния организма студентов высших учебных заведений с ОВ3;

3. Проследить динамику изменений функциональных показателей в контрольной и экспериментальной группах студентов;

4. Экспериментально обосновать эффективность применения унифицированной методики для функционального состояния организма студентов СМГ.

Все чаще встает вопрос о необходимости поиска новых форм двигательной активности студентов с ОВ3 перед зарубежными и отечественными учеными $[15,16,17,18,19,20]$.

Как правило, физкультурно-оздоровительная работа со студентами СМГ не проводится в университетах, а занятия по физической культуре проходят однообразно. Такие настольные игры, как: жульбак, корнхол, кульбутто, новус, шаффлборд являются хорошим инструментов для реше- 
ния данной проблематики: студент с ОВ3, обладая определенными навыками игры, может на равных противостоять сопернику без ограничений в состоянии здоровья и даже одержать победу.

Применение данных игр увеличивает двигательную активность, повышая мотивацию и интерес к занятиям физической культурой, что непосредственно сказывается на физиологических показателях организма студентов.

В нашем исследовании мы предложили студентам заниматься по унифицированной методике, которая включает в себя достаточное количество игр и игровых упражнений за счет использования спортивных настольных игр на занятиях физической культурой. Неоспоримым преимуществом спортивных настольных игр является тот факт, что их можно использовать при разнообразных патологиях у студентов.

\section{Материалы и методы исследования}

При внедрении унифицированной методики произошло увеличение количества игр и игровых упражнений на занятиях физической культурой на $50 \%$. Увеличение количества времени, отведенное на занятия партерной гимнастикой на $20 \%$. Возросло количество специальных беговых упражнений на $10 \%$.

Разработанная методика основывалась на применении настольных спортивных игр, таких как: жульбак, корнхол, кульбутто, новус, шаффлборд.

В исследовании приняли участие 406 студентов (17-20 лет). (Таб. 1)

Таблица 1.

Количественный состав студентов, принявших участие в исследовании

\begin{tabular}{|c|c|c|c|c|c|}
\hline & \multicolumn{2}{|c|}{ Юноши } & \multicolumn{2}{c|}{ Девушки } & \multirow{2}{*}{ Итого } \\
\cline { 2 - 5 } & Эксп. группа & Контр. группа & Эксп. группа & Контр. группа & \\
\hline 1 курс & 43 & 43 & 60 & 60 & 206 \\
\hline 2 курс & 40 & 40 & 60 & 60 & 200 \\
\hline
\end{tabular}

Основные патологические отклонения в состоянии здоровья у студентов специальных медицинских групп:

1. Сердечно-сосудистой системы - 27\% (110 чел.)

2. Органов дыхания $-21 \%$ (85 чел.)

3. Органов слуха $-4 \%$ (16 чел.)

4. Нервной системы - $2 \%$ (8 чел.)

5. Зрения $-15 \%$ (61 чел.)

6. Мочеполовой системы - 3\% (12 чел.) 
7. Эндокринной системы и обмен веществ - $12 \%$ (49 чел.)

8. Опорно-двигательного аппарата - 16\% (65 чел.)

Большинство студентов, относящихся к СМГ, имеют несколько видов нарушений. В этом случае расчет производился по основному прогрессирующему заболеванию.

Для тестирования функционального состояния организма студентов мы провели следующие пробы:

- Проба Руфье (ИР);

- Индекс массы тела (ИМТ кг/м²);

Проба Руфье (ИР) позволяет оценить адаптацию ССС к физическим нагрузкам. Содержание теста: испытуемый в течение 5 минут находится в спокойном состоянии в положении сидя, затем у него подсчитывается ЧСС за 15 секунд (Р1). После этого испытуемый в течение 45 секунд выполняет 30 приседаний. Сразу после приседаний подсчитывается пульс за первые 15 секунд (Р2), а также за последние 15 секунд (Р3) первой минуты отдыха. Затем вычисляется индекс Руфье по формуле: ИР $=(4 \cdot(\mathrm{P} 1+\mathrm{P} 2+\mathrm{P} 3)-200) / 10$.

Оценка результатов теста Руфье:

Менее 0 - отлично (атлетическое сердце); от 0,1 до 5 - очень хорошо; от 5,1 до 10 - хорошо; от 10,1 до 15 - удовлетворительно (сердечная недостаточность средней степени); от 15,1 до 20 - плохо (сердечная недостаточность сильной степени).

Индекс массы тела (ИМТ кг/M²) определялся по формуле: I = P / L2, где P - масса тела (кг), L - длина тела (м).

В соответствии с рекомендациями ВОЗ разработана следующая интерпретация показателей ИМТ:

16 и менее - Выраженный дефицит массы тела

16-18,5 - Недостаточная (дефицит) масса тела

18,5-25 - Норма

25-30 - Избыточная масса тела (предожирение)

30-35 - Ожирение

35-40 - Ожирение резкое

40 и более - Очень резкое ожирение

Описательная статистика результатов исследования представлена:

1 - средней арифметической и ее стандартной ошибкой;

2 - процентными значениями прироста показателя по итогам 3 этапов эксперимента;

3 - U-критерию Манна-Уитни, предназначенного для оценки статистической значимости различий между двумя выборками. 
В связи с тем, что большинство показателей уже на 2 этапе переходят критическое значение при уровне значимости $\mathrm{p}<0,05$, то за минимальный уровень статистической значимости различий между двумя группами приняли $\mathrm{p}<0,01$.

\section{Результаты исследования}

Для более фундаментального педагогического эксперимента данные функциональных проб изучены в динамике и выявлен прирост показателей результатов в процентном соотношении. (Таб. 2, 3)

Таблица 2.

Динамика измерений результатов по Пробе Руфье (Индекс Руфье) у студентов, отнесенных к специальной медицинской группе

\begin{tabular}{|c|c|c|c|c|c|c|c|c|}
\hline 䒘 & 蛋 & $\begin{array}{l}1 \text { этап } \\
\bar{x}_{1} \pm m_{1}\end{array}$ & $\begin{array}{c}2 \text { этап } \\
\bar{x}_{2} \pm m_{2}\end{array}$ & $\begin{array}{l}3 \text { этап } \\
\bar{x}_{3} \pm m_{3}\end{array}$ & $\begin{array}{l}\text { U эмп. } \\
1 \text { эт. } \\
\text { P (U } \\
\text { кр.) }\end{array}$ & $\begin{array}{l}\text { U эмпп. } \\
2 \text { эт. } \\
\text { P (U } \\
\text { кр.) }\end{array}$ & $\begin{array}{c}\text { U эмп. } \\
3 \text { эт. } \\
\text { P (Uкр.) }\end{array}$ & 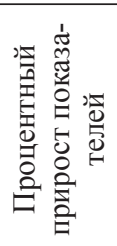 \\
\hline \multirow{2}{*}{ 总貝 } & $Э$ & $\begin{array}{c}11,07 \pm \\
0,59\end{array}$ & $\begin{array}{c}9,16 \pm \\
0,56\end{array}$ & $\begin{array}{c}6,44 \pm \\
0,48\end{array}$ & \multirow{2}{*}{$\begin{array}{c}866,5 \\
P>0,05 \\
(733)\end{array}$} & \multirow{2}{*}{$\begin{array}{c}693 \\
P>0,01 \\
(654)\end{array}$} & \multirow{2}{*}{$\begin{array}{c}438,5 \\
\mathrm{P}<0,01 \\
(654)\end{array}$} & $-41,8$ \\
\hline & К & $\begin{array}{c}10,65 \pm \\
0,58 \\
\end{array}$ & $\begin{array}{c}10,58 \pm \\
0,58 \\
\end{array}$ & $\begin{array}{c}10,37 \pm \\
0,58 \\
\end{array}$ & & & & $-2,6$ \\
\hline \multirow{2}{*}{ 总罾 } & Э & $\begin{array}{c}13,3 \pm \\
0,44 \\
\end{array}$ & $\begin{array}{c}11,4 \pm \\
0,4 \\
\end{array}$ & $\begin{array}{c}8,35 \pm \\
0,34 \\
\end{array}$ & \multirow{2}{*}{$\begin{array}{c}1734 \\
P>0,05 \\
(1486)\end{array}$} & \multirow{2}{*}{$\begin{array}{c}1266 \\
P<0,01 \\
(1356)\end{array}$} & \multirow{2}{*}{$\begin{array}{c}576 \\
\\
\mathrm{P}<0,01 \\
(1356)\end{array}$} & $-37,2$ \\
\hline & K & $\begin{array}{c}13,15 \pm \\
0,42\end{array}$ & $\begin{array}{c}12,88 \pm \\
0,41\end{array}$ & $\begin{array}{c}12,46 \pm \\
0,39\end{array}$ & & & & $-5,2$ \\
\hline \multirow{2}{*}{ 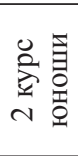 } & Э & $\begin{array}{c}11,47 \pm \\
0,62 \\
\end{array}$ & $\begin{array}{c}9,42 \pm \\
0,56\end{array}$ & $\begin{array}{c}6,47 \pm \\
0,47 \\
\end{array}$ & \multirow{2}{*}{$\begin{array}{c}719,5 \\
P>0,05 \\
(628)\end{array}$} & \multirow{2}{*}{$\begin{array}{c}654.5 \\
P>0,05 \\
(628)\end{array}$} & \multirow{2}{*}{$\begin{array}{c}336.5 \\
\\
\mathrm{P}<0,01 \\
(557)\end{array}$} & $-43,6$ \\
\hline & K & $\begin{array}{c}10,7 \pm \\
0,55 \\
\end{array}$ & $\begin{array}{c}10,57 \pm \\
0,55 \\
\end{array}$ & $\begin{array}{c}10,35 \pm \\
0,55 \\
\end{array}$ & & & & $-3,3$ \\
\hline \multirow{2}{*}{ 总兽 } & $Э$ & $\begin{array}{c}14,13 \pm \\
0,41 \\
\end{array}$ & $\begin{array}{c}12,1 \pm \\
0,38 \\
\end{array}$ & $\begin{array}{c}8,97 \pm \\
0,35 \\
\end{array}$ & \multirow{2}{*}{$\begin{array}{c}1700 \\
P>0,05 \\
(1486)\end{array}$} & \multirow{2}{*}{$\begin{array}{c}975 \\
\\
P<0,01 \\
(1356)\end{array}$} & \multirow{2}{*}{$\begin{array}{c}372 \\
P<0,01 \\
(1356)\end{array}$} & $-36,5$ \\
\hline & К & $\begin{array}{c}14,46 \pm \\
0,39 \\
\end{array}$ & $\begin{array}{c}14,37 \pm \\
0,39 \\
\end{array}$ & $\begin{array}{c}14,21 \pm \\
0,38\end{array}$ & & & & $-1,73$ \\
\hline
\end{tabular}

Сравнив результаты на каждом этапе в рамках двух групп - контрольной и экспериментальной, можно наблюдать отсутствие различий на первом этапе и появление их на третьем. 
По результатам исследования пробы Руфье у юношей и девушек экспериментальной группы наблюдается значительный прирост результатов, в отличие от контрольной. Это доказывает, что применяемая унифицированная методика положительно влияет на состояние сердечно-сосудистой системы студентов, отнесенных к СМГ.

Таблийа 3.

Динамика измерений результатов ИМТ (кг/м²) у студентов, отнесенных к специальной медицинской группе

\begin{tabular}{|c|c|c|c|c|c|c|c|c|}
\hline 龸 & 胥 & $\begin{array}{l}1 \text { этап } \\
\bar{x}_{1} \pm m_{1}\end{array}$ & $\begin{array}{l}2 \text { этап } \\
\bar{x}_{2} \pm m_{2}\end{array}$ & $\begin{array}{l}3 \text { этап } \\
\bar{x}_{3} \pm m_{3}\end{array}$ & $\begin{array}{c}\text { U эмп. } \\
1 \text { эт. } \\
\text { P (U } \\
\text { кр.) }\end{array}$ & $\begin{array}{c}\text { U эмп. } \\
2 \text { эт. } \\
\text { P (U } \\
\text { кр.) }\end{array}$ & $\begin{array}{c}\text { U эмп. } \\
3 \text { эт. } \\
\text { P (U } \\
\text { кр.) }\end{array}$ & 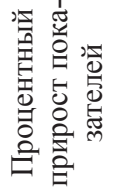 \\
\hline \multirow{2}{*}{ 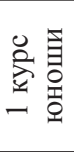 } & $Э$ & $\begin{array}{c}26,91 \pm \\
0,63\end{array}$ & $\begin{array}{c}25,48 \pm \\
0,47\end{array}$ & $\begin{array}{c}23,58 \pm \\
0,28\end{array}$ & \multirow{2}{*}{$\begin{array}{c}884 \\
P>0,05 \\
(733)\end{array}$} & \multirow{2}{*}{$\begin{array}{c}724.5 \\
P>0,01 \\
(654)\end{array}$} & \multirow{2}{*}{$\begin{array}{c}532 \\
P<0,01 \\
(654)\end{array}$} & $-12,4$ \\
\hline & $\mathrm{K}$ & $\begin{array}{c}26,75 \pm \\
0,62\end{array}$ & $\begin{array}{c}26,55 \pm \\
0,6\end{array}$ & $\begin{array}{c}25,94 \pm \\
0,54\end{array}$ & & & & -3 \\
\hline \multirow{2}{*}{ 总學 } & $Э$ & $\begin{array}{c}25,85 \pm \\
0,6\end{array}$ & $\begin{array}{c}24,26 \pm \\
0,45\end{array}$ & $\begin{array}{c}22,43 \pm \\
0,29\end{array}$ & \multirow{2}{*}{$\begin{array}{c}1698 \\
P>0,05 \\
(1486)\end{array}$} & \multirow{2}{*}{$\begin{array}{l}1368.5 \\
P>0,01 \\
(1356)\end{array}$} & \multirow{2}{*}{$\begin{array}{l}989.5 \\
\mathrm{P}<0,01 \\
(1356)\end{array}$} & $-13,2$ \\
\hline & $\mathrm{K}$ & $\begin{array}{c}26,38 \pm \\
0,65\end{array}$ & $\begin{array}{c}26,2 \pm \\
0,62 \\
\end{array}$ & $\begin{array}{c}25,87 \pm \\
0,59\end{array}$ & & & & $-1,9$ \\
\hline \multirow{2}{*}{ 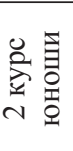 } & Э & $\begin{array}{c}26,82 \pm \\
0,56 \\
\end{array}$ & $\begin{array}{c}25,19 \pm \\
0,38\end{array}$ & $\begin{array}{c}23,28 \pm \\
0,22\end{array}$ & \multirow{2}{*}{$\begin{array}{c}773.5 \\
P>0,05 \\
(628)\end{array}$} & \multirow{2}{*}{$\begin{array}{c}609 \\
P>0,01 \\
(557)\end{array}$} & \multirow{2}{*}{$\begin{array}{c}393.5 \\
P<0,01 \\
(557)\end{array}$} & $-13,1$ \\
\hline & $\mathbf{K}$ & $\begin{array}{c}26,59 \pm \\
0,57\end{array}$ & $\begin{array}{c}26,42 \pm \\
0,55\end{array}$ & $\begin{array}{c}26,07 \pm \\
0,51\end{array}$ & & & & -2 \\
\hline \multirow{2}{*}{ 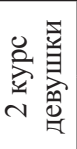 } & $\ni$ & $\begin{array}{c}26,19 \pm \\
0,62\end{array}$ & $\begin{array}{c}24,53 \pm \\
0,47\end{array}$ & $\begin{array}{c}22,57 \pm \\
0,3\end{array}$ & \multirow{2}{*}{$\begin{array}{c}1724.5 \\
P>0,05 \\
(1486)\end{array}$} & \multirow{2}{*}{$\begin{array}{l}1402 \\
P>0,01 \\
(1356)\end{array}$} & \multirow{2}{*}{$\begin{array}{c}1041 \\
P<0,01 \\
(1356)\end{array}$} & $-13,8$ \\
\hline & $\mathbf{K}$ & $\begin{array}{c}26,63 \pm \\
0,68\end{array}$ & $\begin{array}{c}26,44 \pm \\
0,66\end{array}$ & $\begin{array}{c}26,08 \pm \\
0,63\end{array}$ & & & & $-2,1$ \\
\hline
\end{tabular}

В результате оценки ИМТ при сравнении результатов контрольной и экспериментальной группы на 1 этапе не наблюдается статистическая значимость различий. На 2 этапе некоторые показатели переходят критические значения при уровне статистической значимости различий $\mathrm{p}<0,05$, но не достигают уровня при $p<0,01$. На 3 этапе наблюдается более значительный прирост функциональных способностей организма студентов, как следствие - все показатели уверенно входят в зону значимости при уровне $\mathrm{p}<0,01$. Прирост процентного показателя значительно выше у экспериментальной группы, чем у контрольной. 


\section{Обсуждение}

На протяжении 1 этапа результаты не являются статистически значимыми, что показывает однородность сравниваемых групп в начале эксперимента. На 3 этапе все показатели являются статистически значимыми. Это доказывает эффективность применяемой методики даже при высокой чувствительности U-критерия Манна-Уитни.

В экспериментальных группах наблюдается явный прирост показателей по средней арифметической. Прирост процентного показателя также значительно выше в экспериментальных группах, чем в контрольных.

Динамика показателей в экспериментальных группах юношей и девушек 1 и 2 курсов показала эффективность применяемой унифицированной методики в части снижения веса и положительного влияния на состояние сердечно-сосудистой системы за счет увеличения двигательной активности.

Согласно анализу полученных данных, прирост показателей, в процентном соотношении экспериментальной группы улучшается более $30 \%$ в показателе Индекса Руфье, более 9\% в показателе ИМТ, по сравнению с контрольной, что позволяет утверждать об эффективности применяемой методики.

\section{Заключение}

В ходе проведенного исследования были изучены показатели функционального состояния организма студентов высших учебных заведений с OB3. Большинство показателей Индекса Руфье и ИМТ на 1 этапе эксперимента имеют значимые отклонения от нормы.

Согласно анализу полученных данных статистическая значимость различий выявлена на третьем этапе между контрольной и экспериментальной группой у юношей и девушек 1 и 2 курсов. Положительная динамика изменений функциональных показателей экспериментальной группы позволяет сделать вывод о том, что значительно улучшилось физиологическое состояние студентов, отнесенных к СМГ.

Таким образом, проведенное исследование показало, что примененная методика подходит студентам с любыми видами отклонений в состоянии здоровья и улучшает прирост функциональных показателей организма. Данная унифицированная методика является хорошим средством здоровьесбережения и отвечает всем требованиям инклюзивного образования.

Информация о конфликте интересов. Конфликты интересов отсутствуют. 
Благодарности. Авторы выражают благодарность анонимным рецензентам за их труд и ценные предложения, высказанные по поводу содержания статьи.

\section{Список литературы}

1. María Martín-Garcíaa, Luis M. Alegrea, Beatriz García-Cuartero, Eleanor J. Bryant, Bernard Gutin, Ignacio Ara, Effects of a 3-month vigorous physical activity intervention on eating behaviors and body composition in overweight and obese boys and girls // Journal of Sport and Health Science, Volume 8, Issue 2, March 2019. P. 170-176. https://doi.org/10.1016/j.jshs.2017.09.012

2. Голубятникова М.В., Яковлева В.Н., Макарова Л.Н., Агеева М.В. Влияние физических упражнений на показатели коэффициента здоровья, физическую подготовленность, физическое состояние и работоспособность студентов в процессе занятий физической культурой // Спортивная медицина: наука и практика. 2017. Т. 7. № 3. С. 14-21.

3. Mohammadi E., \&Saberi A. The relationship between body composition, anthropometry, and physical fitness in female university students // TSS, 2016, 3(23), P. 155-158.

4. Bray S.R., \&Born, H.A. Transition to university and vigorous physical activity: implications for health and psychological well-being during transition to university life // J Am Coll Health. 2004. № 52, P. 11-15. https://pubmed.ncbi. nlm.nih.gov/15018429/

5. Pistlova L., Balint G., \&Sedlacek J. Physical development and general motor performance of Bratislava university students // Procedia - Social and Behavioral Sciences. 2014. P. 234-246.

6. Чоговадзе А.В., Рыжак М.М. Физическое воспитание и формирование здорового образа жизни студентов // Теория и практика физической культуры. 1993, № 7. C. 4.

7. Block M.E., \& Obrusnikova I. What is Inclusion? In M. E. Block (Ed.), A Teacher's Guide to Adapted Physical Education (4th ed.), Baltimore (MD): Paul H. Brookes. 2016. P. 19-34.

8. Cordente D., González S., Block M. E., \& Contreras, O. Structure, validity and reliability of the Children's Attitudes Towards Integrated Physical EducationSpanish version (CAIPE-SP) // European Journal of Adapted Physical Activity. 2016, 9(2), P. 3-12.

9. Ruscitti R., Thomas S.G., \& Bentley D. The experiences of students without disabilities in inclusive physical education classrooms: a review of literature // Asia-Pacific Journal of Health, Sport and Physical Education. 2017, 8(3). P. 741-747. doi.org/10.1080/18377122.2017.1345286 
10. McKay C., Block M., \& Park J. Y. The Impact of Paralympic School Day on Student Attitudes Toward Inclusion in Physical Education // Adapted Physical Activity Quarterly. 2016, 32. P. 331-348. doi: 10.1123/APAQ.2015-0045

11. Катриш Е.В. О проблеме инклюзивного образования в сфере физической культуры // Сибирский педагогический журнал. 2015, №3. С.121-124.

12. Минёнок Е.В., Лутковская О.Ю. Адаптивное физическое воспитание как средство укрепления здоровья студентов специальных медицинских групп // Вестник Полоцкого государственного университета. Серия Е. Педагогические науки. 2019. № 15. С. 159-162.

13. Готовцев Е.А., Германов Г.Н., Романова Ю.В., Машошина И.В. Мониторинг состояния здоровья и физической подготовленности студентов как методология анализа и оценки продуктивности процесса физического воспитания // Ученые записки университета им. П.Ф. Лесгафта. 2012. № 1 (83). С. 40-45.

14. Зайцев П.В. Контроль за динамикой состояния здоровья и двигательной активности студентов / П.В. Зайцев, В.Ф. Стафеев, А.М. Образцова // Вопросы физического воспитания студентов. Л.: ДГУ, 1991. Вып. 22. С.6.

15. Gebhardt D. L., \& Baker T. A. Physical performance. In J. C. Scott \& D. H. Reynolds (Eds.). Handbook of workplace assessment. San Francisco, CA: Jossey-Bass. 2010. P. 143-151.

16. Marko M., Rozim R., Kurková P., Bendíková E. Level of students posture in adolescence in relation to physical activity // European Journal of Physical Education and Sport. 2018. № 6-2. P. 61-67.

17. Лубышева Л.И., Грузных Г.М. Теоретико-методические обоснования физического воспитания студентов // Теория и практика физ. культуры. 1991, № 6. С. 4.

18. Манжелей И.В. Концептуальные основы дифференцированного физкультурного образования студентов системы среднего профессионального образования // Теория и практика физической культуры. 2001, № 3. С. 22.

19. Рипа М.Д., Кулькова И.В. Лечебно-оздоровительные технологии в адаптивном физическом воспитании: учебное пособие для вузов. 2-е изд., испр. и доп. Москва : Издательство Юрайт, 2020. С. 46.

20. Лукина С.М., Лобанов Ю.Я., Шаронова А.В., Ярчиковская Л.В., Миронова О.В. Освоение дисциплины «Физическая культура и спорт» в ВУЗе с применением инновационных технологий // Теория и практика физической культуры. 2019. № 4. С. 44-46.

\section{References}

1. María Martín-Garcíaa, Luis M. Alegrea, Beatriz García-Cuartero, Eleanor J. Bryant, Bernard Gutin, Ignacio Ara, Effects of a 3-month vigorous physical 
activity intervention on eating behaviors and body composition in overweight and obese boys and girls. Journal of Sport and Health Science, Volume 8, Issue 2, March 2019. P. 170-176. https://doi.org/10.1016/j.jshs.2017.09.012

2. Golubyatnikova M.V., Yakovleva V.N., Makarova L.N., Ageeva M.V. Vliyanie fizicheskikh uprazhneniy na pokazateli koeffitsienta zdorov'ya, fizicheskuyu podgotovlennost', fizicheskoe sostoyanie i rabotosposobnost' studentov v protsesse zanyatiy fizicheskoy kul'turoy [The influence of physical exercises on indicators of the coefficient of health, physical fitness, physical condition and working capacity of students in the process of physical education]. Sportivnaya meditsina: nauka i praktika [Sports medicine: science and practice]. 2017. Vol. 7. № 3. P. 14-21.

3. Mohammadi E., \& Saberi A. The relationship between body composition, anthropometry, and physical fitness in female university students. TSS, 2016, 3 (23), P. 155-158.

4. Bray S.R., \& Born, H.A. Transition to university and vigorous physical activity: implications for health and psychological well-being during transition to university life. J Am Coll Health. 2004. No. 52, P. 11-15. https://pubmed. ncbi.nlm.nih.gov/15018429/

5. Pistlova L., Balint G., \& Sedlacek J. Physical development and general motor performance of Bratislava university students. Procedia -Social and Behavioral Sciences. 2014. P. 234-246.

6. Chogovadze A.B., Ryzhak M.M. Fizicheskoe vospitanie i formirovanie zdorovogo obraza zhizni studentov [Physical education and the formation of a healthy lifestyle of students]. Teoriya i praktika fizicheskoy kul'tury [Theory and practice of physical culture]. 1993, № 7. P. 4.

7. Block M. E., \& Obrusnikova I. What is Inclusion? In M. E. Block (Ed.), A Teacher's Guide to Adapted Physical Education (4th ed.), Baltimore (MD): Paul H. Brookes. 2016. P. 19-34.

8. Cordente D., González S., Block M. E., \& Contreras, O. Structure, validity and reliability of the Children's Attitudes Towards Integrated Physical Education-Spanish version (CAIPE-SP). European Journal of Adapted Physical Activity. 2016, 9 (2), P. 3-12.

9. Ruscitti R., Thomas S. G., \& Bentley D. The experiences of students without disabilities in inclusive physical education classrooms: a review of literature. Asia-Pacific Journal of Health, Sport and Physical Education. 2017, 8 (3). P. 741-747. doi.org/10.1080/18377122.2017.1345286

10. McKay C., Block M., \& Park J. Y. The Impact of Paralympic School Day on Student Attitudes Toward Inclusion in Physical Education. Adapted Physical Activity Quarterly. 2016, 32. P. 331-348. doi: 10.1123/APAQ.2015-0045 
11. Katrish E.V. O probleme inklyuzivnogo obrazovaniya v sfere fizicheskoy kul'tury [On the problem of inclusive education in the field of physical culture]. Sibirskiy pedagogicheskiy zhurnal [Siberian pedagogical journal]. 2015, no. 3. P. 121-124.

12. Minenok E.V., Lutkovskaya O.Yu. Adaptivnoe fizicheskoe vospitanie kak sredstvo ukrepleniya zdorov'ya studentov spetsial'nykh meditsinskikh grupp [Adaptive physical education as a means of strengthening the health of students of special medical groups]. Vestnik Polotskogo gosudarstvennogo universiteta. Seriya E. Pedagogicheskie nauki [Bulletin of Polotsk State University. Series E. Pedagogical Sciences]. 2019. № 15. P. 159-162.

13. Gotovtsev E.A., Germanov G.N., Romanova Yu.V., Mashoshina I.V. Monitoring sostoyaniya zdorov'ya i fizicheskoy podgotovlennosti studentov kak metodologiya analiza i otsenki produktivnosti protsessa fizicheskogo vospitaniya [Monitoring of the state of health and physical fitness of students as a methodology for analyzing and assessing the productivity of the process of physical education]. Uchenye zapiski universiteta im. P.F. Lesgafta. 2012. № 1 (83). P. 40-45.

14. Zaitsev P.V., Stafeev V.F., Obraztsova A.M. Kontrol' za dinamikoy sostoyaniya zdorov'ya i dvigatel'noy aktivnosti studentov [Control over the dynamics of the state of health and motor activity of students]. Voprosy fizicheskogo vospitaniya studentov [Questions of physical education of students]. L.: DGU, 1991. Issue 22. P. 6.

15. Gebhardt D. L., \& Baker T. A. Physical performance. In J. C. Scott \& D. H. Reynolds (Eds.). Handbook of workplace assessment. San Francisco, CA: Jossey - Bass. 2010. P. 143-151.

16. Marko M., Rozim R., Kurková P., Bendíková E. Level of students posture in adolescence in relation to physical activity. European Journal of Physical Education and Sport. 2018. № 6-2. P. 61-67.

17. Lubysheva L.I., Gruznykh G.M. Teoretiko-metodicheskie obosnovaniya fizicheskogo vospitaniya studentov [Theoretical and methodological substantiation of physical education of students]. Teoriya i praktika fizicheskoy kul'tury [Theory and practice of physical culture]. 1991, № 6. P. 4.

18. Manzheley I.V. Kontseptual'nye osnovy differentsirovannogo fizkul'turnogo obrazovaniya studentov sistemy srednego professional'nogo obrazovaniya [Conceptual foundations of differentiated physical education of students of the system of secondary vocational education]. Teoriya i praktika fizicheskoy kul'tury [Theory and practice of physical culture]. 2001, № 3. P. 22.

19. Ripa M.D., Kulkova I.V. Lechebno-ozdorovitel'nye tekhnologii v adaptivnom fizicheskom vospitanii [Health-improving technologies in adaptive 
physical education]: textbook for universities. 2nd ed., Rev. and add. Moscow: Yurayt Publishing House, 2020, p. 46.

20. Lukina S.M., Lobanov Yu.Ya., Sharonova A.V., Yarchikovskaya L.V., Mironova O.V. Osvoenie distsipliny «Fizicheskaya kul'tura i sport»v VUZe s primeneniem innovatsionnykh tekhnologiy [Mastering the discipline "Physical culture and sport" in the university with the use of innovative technologies]. Teoriya i praktika fizicheskoy kul'tury [Theory and practice of physical culture]. 2019. № 4. P. 44-46.

\section{ДАННЫЕ ОБ АВТОРАХ}

Козлов Алексей Владимирович, диссертант, старший преподаватель, кафедра «Физическая культура и спорт»

ФГБОУ ВО «Московский государственный университет пищевых производств»

Волоколамское шоссе, 11, г. Москва, 125080, Российская Федераиия 19880588@bk.ru

Каткова Анастасия Михайловна, кандидат педагогических наук, доцент, заведующая кафедрой «Физическая культура и спорт»

ФГБОУ ВО «Московский государственный университет пищевых производств»

Волоколамское шоссе, 11, г. Москва, 125080, Российская Федерация

katkova@mgupp.ru

Козлова Мария Александровна, кандидат педагогических наук, доцент, кафедра «Физическая культура и спорт»

ФГБОУ ВО «Московский государственный университет пищевых производств»

Волоколамское шоссе, 11, г. Москва, 125080, Российская Федераиия 7012946@bk.ru

\section{DATA ABOUT THE AUTHORS}

Kozlov Alexey Vladimirovich, Dissertator, Senior lecturer, Department of Physical Culture and Sports Moscow State University of Food Production

11, Volokolamskoe highway, Moscow, 125080, Russian Federation 19880588@bk.ru 
ORCID: 0000-0001-6347-7007

SPIN-code: 4935-4109

Katkova Anastasia Mikhailovna, Candidate of Pedagogical Sciences, Associate Professor, Head of the Department of Physical Culture and Sports Moscow State University of Food Production

11, Volokolamskoe highway, Moscow, 125080, Russian Federation

katkova@mgupp.ru

ORCID: 0000-0003-1630-0115

SPIN-code: 2497-1326

Kozlova Maria Alexandrovna, Candidate of Pedagogical Sciences, Associate Professor, Department of Physical Culture and Sports Moscow State University of Food Production

11, Volokolamskoe highway, Moscow, 125080, Russian Federation 7012946@bk.ru

ORCID: 0000-0003-4138-0903 